\title{
Relation between nonlinear refractive index and third-order susceptibility in absorbing media
}

\author{
Raúl del Coso and Javier Solis \\ Instituto de Optica, Consejo Superior de Investigaciones Científicas, Serrano 121, 28006, Madrid, Spain
}

Received September 12, 2003; revised manuscript received November 6, 2003; accepted November 10, 2003

Expressions relating complex third-order optical susceptibility $\left(\chi^{(3)}=\chi_{R}^{(3)}+i \chi_{I}^{(3)}\right)$ with nonlinear refractive index $\left(n_{2}\right)$ and nonlinear absorption coefficient $(\beta)$ have been formulated that eliminate the commonly used approximation of a negligible linear absorption coefficient. The resulting equations do not show the conventional linear dependence of $\chi_{R}^{(3)}$ with $n_{2}$ and $\chi_{I}^{(3)}$ with $\beta$. Nonlinear refraction and absorption result instead from the interplay between the real and imaginary parts of the first- and third-order susceptibilities of the material. This effect is illustrated in the case of a metal-dielectric nanocomposite for which $n_{2}$ and $\beta$ values were experimentally obtained by Z-scan measurements and for which the use of the new formulas for $\chi_{R}^{(3)}$ and $\chi_{I}^{(3)}$ yield a large correction and a sign reversal for $\chi_{I}^{(3)}$. (C) 2004 Optical Society of America

OCIS codes: $190.0190,190.3270,190.4400$.

\section{INTRODUCTION}

Third-order nonlinear optical effects are the physical basis of a number of applications in future high-capacity communication networks ${ }^{1}$ in which ultrafast switching, signal regeneration, and high-speed demultiplexing would be performed all-optically through the use of thirdorder optical nonlinearities. Thus, in recent years, thirdorder nonlinearities have been studied experimentally in a large variety of artificial materials, which include homogeneous bulk glasses and polymers ${ }^{2,3}$ and nano- and mesoscopically structured materials such as semiconductor, quantum-well structures ${ }^{4}$ or glasses doped with either semiconductor $^{5}$ or metal nanocrystals. ${ }^{6}$ Optimization of the design of these materials for a given application depends, to a large extent, on comprehension of the physical mechanisms responsible for the nonlinear response. In this context, it is important to remark that the real and imaginary parts of $\chi^{(3)}$ are the physical parameters that contain the information relative to the microscopic origin of the nonlinearity since they are related to the hyperpolarizabilities of the system. ${ }^{7,8}$ However, these quantities are not directly measurable in most cases. Instead, they are obtained through experimental methods that are sensitive to other macroscopic manifestations of $\chi^{(3)}$, such as phase modulation, nonlinear refraction, wave mixing and nonlinear absorption. ${ }^{9}$

In this work we report a general and simple determination of the relation between the real and imaginary parts of the third-order susceptibility and the nonlinear refractive index and absorption coefficient that is valid for absorbing systems. The most interesting consequence of the expressions obtained is that nonlinear refraction and absorption are no longer directly related to the real and imaginary parts of the third-order susceptibility, but are a consequence of the interplay between the real and imaginary parts of the first- and third-order susceptibilities of the system. This gives rise to the appearance, for instance, of nonlinear refraction effects in systems with a purely imaginary susceptibility or, on the other hand, to the appearance of measurable nonlinear absorption effects in systems with a real third-order susceptibility. The expressions obtained still permit one to determine analytically the sign and magnitude of the third-order susceptibility components, thereby avoiding the errors that appear (particularly in the sign of the components) when applying the usual expression (which assumes negligible absorption) to absorbing materials. The new formulas have been applied to the case of a real absorbing material with Kerr-type nonlinearity, a metal-dielectric nanocomposite whose nonlinear refractive index and absorption coefficient were experimentally determined by Z-scan experiments.

\section{FORMULAS RELATING $n_{2}, \beta$, AND $\chi^{(3)}$}

In the case of Kerr-type nonlinearities, the expressions relating the refractive index and the absorption coefficient with the intensity $I$ of the electromagnetic wave are $n=n_{0}+n_{2} I$ and $\alpha=\alpha_{0}+\beta I$, where $n_{0}$ and $\alpha_{0}$ are the linear refractive index and linear absorption coefficient, respectively.

In a system showing a negligible absorption $\left(\alpha_{0} \approx 0\right)$, the nonlinear refractive index $n_{2}$ and the nonlinear absorption coefficient $\beta$ are proportional to the real $\chi_{R}^{(3)}$ and imaginary $\chi_{I}^{(3)}$ parts of $\chi^{(3)}$ through the following expressions in the international system (SI) of units ${ }^{9}$ :

$$
\begin{aligned}
& \chi_{R}^{(3)}=(4 / 3) n_{0}{ }^{2} \epsilon_{0} c n_{2}, \\
& \chi_{I}^{(3)}=\left(n_{0}{ }^{2} \epsilon_{0} c \lambda / 3 \pi\right) \beta,
\end{aligned}
$$

where $n_{0}, \epsilon_{0}$, and $\lambda$ denote, respectively, the linear refractive index of the material, the electric permittivity of free space $\left(8.85 \times 10^{-12} \mathrm{~F} / \mathrm{m}\right)$, and the wavelength. Under this restriction of negligible linear absorption, it is clear that a purely real third-order susceptibility will manifest itself through purely refractive nonlinear ef- 
fects, whereas a purely imaginary susceptibility will lead only to nonlinear absorption effects.

In spite of the assumption of negligible absorption implicit in Eqs. (1), they have been used very often ${ }^{10,11}$ for finding the values of $\chi_{R}^{(3)}$ and $\chi_{I}^{(3)}$ in absorbing systems $(k \neq 0)$, particularly since Z-scan measurements ${ }^{10,12}$ became one of the most popular methods for the assessment of third-order nonlinearities. Formulas similar to Eqs. (1) are also quite commonly applied to absorbing materials in the field of near-resonance, nonlinear spectroscopy. ${ }^{13,14}$ In what follows we will derive a set of expressions relating $\chi_{R}^{(3)}, \chi_{I}^{(3)}, n_{2}$, and $\beta$ valid for absorbing systems.

By following Ref. 8, the nonlinear response of a material system under an applied external electromagnetic field can be described by a series expansion of the polarization as

$$
\widetilde{P}=\epsilon_{0}\left[\tilde{\chi}^{(1)} \widetilde{E}+\tilde{\chi}^{(2)} \tilde{E} \widetilde{E}+\tilde{\chi}^{(3)} \widetilde{E} \widetilde{E} \widetilde{E}^{*}+\ldots\right],
$$

where $\widetilde{P}$ denotes the complex polarization vector, $\widetilde{\chi}^{(n)}$ corresponds to the $n$ th-order complex susceptibility tensor, and $\widetilde{E}$ denotes the complex electric field.

The complex displacement vector $\widetilde{D}$ is related to the polarization and the electric field through the expression (using SI units):

$$
\widetilde{D}=\epsilon_{0} \widetilde{E}+\widetilde{P}=\epsilon_{0}(1+\widetilde{\chi}) \widetilde{E}=\epsilon \widetilde{E} .
$$

In centrosymmetric materials, by neglecting wavemixing, second-order effects, the susceptibility $\tilde{\chi}$ up to the third order corresponds to

$$
\tilde{\chi}=\tilde{\chi}^{(1)}+\tilde{\chi}^{(3)} \widetilde{E} \widetilde{E}^{*},
$$

which in the case of a material under a single, monochromatic, linearly polarized wave can be reduced to the scalar expression ${ }^{9}$

$$
\chi(\omega)=\chi^{(1)}(\omega)+3 \chi^{(3)}(\omega)|E|^{2},
$$

where $\chi^{(3)}(\omega)$ now denotes the complex component $\chi_{i i i i}^{(3)}(\omega ; \omega, \omega,-\omega)$ of the third-order susceptibility tensor and $|E|$ denotes the amplitude of the electric field.

To determine the relation between the real and imaginary parts of $\chi^{(3)}$ and the nonlinear refractive index and absorption coefficient, we will use the expressions relating the complex dielectric constant $\left(\epsilon=\epsilon_{1}+i \epsilon_{2}\right)$ and the complex refractive index $(\tilde{n}=n+i k)$ at a given wavelength:

$$
\begin{aligned}
& \epsilon_{1}=\epsilon_{0}\left(n^{2}-k^{2}\right), \\
& \epsilon_{2}=\epsilon_{0}(2 n k) ; \\
& n=\sqrt{\frac{|\epsilon|+\epsilon_{1}}{2 \epsilon_{0}},} \\
& k=\sqrt{\frac{|\epsilon|-\epsilon_{1}}{2 \epsilon_{0}} .}
\end{aligned}
$$

These are the basic formulas necessary to obtain the change in the refractive index $\Delta n$ and the absorption coefficient $\Delta k$ associated with a variation of the dielectric function of the material due to a nonlinear polarization response. By using relations (3) and (5) we can express the dielectric function as

$$
\epsilon=\epsilon^{(1)}+\Delta \epsilon,
$$

where $\epsilon^{(1)}=\epsilon_{0}\left(1+\chi^{(1)}\right)$ is the linear dielectric function and $\Delta \epsilon=3 \epsilon_{0} \chi^{(3)}|E|^{2}$ is the nonlinear contribution to $\epsilon$ due to third-order nonlinear polarization of the material. The real and imaginary parts of $\Delta \epsilon$ are given by

$$
\begin{aligned}
& \Delta \epsilon_{1}=3 \epsilon_{0} \chi_{R}^{(3)}|E|^{2}, \\
& \Delta \epsilon_{2}=3 \epsilon_{0} \chi_{I}^{(3)}|E|^{2} .
\end{aligned}
$$

In the case of a Kerr-type nonlinear response we can express the dependence of the refractive index and the absorption coefficient on the intensity of light ${ }^{9}$ $\left(I=2 \epsilon_{0} n_{0} c|E|^{2}\right)$ as

$$
\begin{aligned}
& n=n_{0}+\Delta n=n_{0}+n_{2} I, \\
& \alpha=\alpha_{0}+\Delta \alpha=\alpha_{0}+\beta I,
\end{aligned}
$$

although in our case, it is more practical to express Eq. (10) in terms of the imaginary part of the refractive index $k=\lambda \alpha / 4 \pi$ as

$$
k=k_{0}+\Delta k=k_{0}+k_{2} I,
$$

where $k_{2}=\beta \lambda / 4 \pi$ is included in order to have symmetrical expressions for the nonlinear refractive and absorptive indexes in subsequent equations. By using the expressions (6A), (9) and (11) we obtain that

$$
\begin{aligned}
\epsilon_{1} & =\epsilon_{1}^{(1)}+\Delta \epsilon_{1} \\
& =\epsilon_{0}\left[\left(n_{0}+\Delta n\right)^{2}-\left(k_{0}+\Delta k\right)^{2}\right] \\
& \Rightarrow \Delta \epsilon_{1}=\epsilon_{0}\left[2\left(n_{0} \Delta n-k_{0} \Delta k\right)+(\Delta n)^{2}+(\Delta k)^{2}\right],
\end{aligned}
$$

which, by using the corresponding expressions for $\Delta \epsilon_{1}$ [Eq. (8A)], $\Delta n$ [Eq. (9)], and $\Delta k$ [Eq. (11)], and assuming that $\Delta n \ll n_{0}$ and $\Delta k \ll k_{0}$, leads to

$$
\begin{aligned}
3 \chi_{R}^{(3)}|E|^{2} & =2 I\left(n_{0} n_{2}-k_{0} k_{2}\right) \\
& \Rightarrow \chi_{R}^{(3)}=\left(4 n_{0} \epsilon_{0} c / 3\right)\left(n_{0} n_{2}-k_{0} k_{2}\right) .
\end{aligned}
$$

A similar procedure for the imaginary part of $\epsilon$ leads to

$$
\chi_{I}^{(3)}=\left(4 n_{0} \epsilon_{0} c / 3\right)\left(n_{0} k_{2}+k_{0} n_{2}\right) .
$$

The second-order terms $\Delta n^{2}$ and $\Delta k^{2}$ have been neglected since they become important only in extreme conditions: very high third-order nonlinear effects (large $\chi^{(3)}$ and intensity) and nonnegligible absorption, where other effects such as thermo-optical ones or fifth-order nonlinearities can also become significant.

Both expressions (13) and (14) reduce obviously to the relations given in Eqs. (1) when the absorption of the system is negligible $\left(k_{0} \approx 0\right)$. In absorbing systems, the nonlinear absorption and refraction are thus the consequence of the interplay between the real and imaginary parts of the first- and third-order susceptibilities, and $n_{2}$ and $\beta$ are no longer proportional to the real and imaginary parts of $\chi^{(3)}$. This can be seen more clearly when looking at the inverse expressions obtained from Eqs. (13) and (14): 


$$
\begin{aligned}
& n_{2}=\frac{3}{4 \epsilon_{0} c\left(n_{0}{ }^{2}+k_{0}{ }^{2}\right)}\left[\chi_{R}^{(3)}+\frac{k_{0}}{n_{0}} \chi_{I}^{(3)}\right], \\
& k_{2}=\frac{3}{4 \epsilon_{0} c\left(n_{0}{ }^{2}+k_{0}{ }^{2}\right)}\left[\chi_{I}^{(3)}-\frac{k_{0}}{n_{0}} \chi_{R}^{(3)}\right] ;
\end{aligned}
$$

this latter can be expressed in terms of the usually measured $\beta$ as

$$
\beta=\frac{3 \pi}{\lambda \epsilon_{0} c\left(n_{0}^{2}+k_{0}^{2}\right)}\left[\chi_{I}^{(3)}-\frac{k_{0}}{n_{0}} \chi_{R}^{(3)}\right] .
$$

From expression (15) we can see that actually $n_{2}$ is similar to the value calculated using Eq. (1) (setting $k_{0}=0$ ) with an added correction term to $\chi_{R}^{(3)}$ given by $k_{0} \chi_{I}^{(3)} / n_{0}$. The same applies to $\beta$ with the correction term $-k_{0} \chi_{R}^{(3)} / n_{0}$ added to $\chi_{I}^{(3)}$.

\section{IMPLICATIONS OF THESE FORMULAS IN ABSORBING MEDIA}

One of the most remarkable consequences of expression (15) is that in materials showing a purely imaginary third-order susceptibility $\left(\chi_{R}^{(3)}=0\right)$ and nonnegligible absorption, $n_{2}$ scales as $\left[\left(k_{0} / n_{0}\right) /\left(1+k_{0}{ }^{2} / n_{0}{ }^{2}\right)\right] \chi_{I}^{(3)}$, and therefore, refractive nonlinearities could become observable. Similarly, for a purely real third-order susceptibility $\left(\chi_{I}^{(3)}=0\right), \quad k_{2}$ will scale as $\left[-\left(k_{0} / n_{0}\right) /\right.$ $\left.\left(1+k_{0}{ }^{2} / n_{0}{ }^{2}\right)\right] \chi_{R}^{(3)}$, and nonlinear absorptive effects will become more pronounced as the material absorption increases. An evaluation of the magnitude of the correction to $n_{2}$ introduced by expression (15) in absorbing materials can be obtained from the ratio of the nonlinear refractive index $n_{2}$ calculated using expression (15) and that $n_{2\left(k_{0}=0\right)}$ calculated with the approximation of negligible absorption given by Eqs. (1):

$$
\frac{n_{2}}{n_{2\left(k_{0}=0\right)}}=\left(1+\frac{k_{0}^{2}}{n_{0}^{2}}\right)^{-1}\left[1+\frac{k_{0}}{n_{0}} \frac{\chi_{I}^{(3)}}{\chi_{R}^{(3)}}\right] .
$$

Remarkably, this ratio is dependent only on the ratios of the absorption coefficient and the refractive index $k_{0} / n_{0}$ and the imaginary and real parts of the third order susceptibility $\chi_{I}^{(3)} / \chi_{R}^{(3)}$

Expression (18) helps to clarify when the formulas deduced in this work [Eqs. (13-16)] have to be used instead of Eqs. (1), valid for $k_{0}=0$. This can be seen more clearly in Fig. 1 where the ratio $n_{2} / n_{2\left(k_{0}=0\right)}$ has been plotted as a function of the ratio $k_{0} / n_{0}$ for different values (positive and negative) of the ratio $\chi_{I}^{(3)} / \chi_{R}^{(3)}$.

For the curves corresponding to positive values of $\chi_{I}^{(3)} / \chi_{R}^{(3)}$, the sign of $n_{2} / n_{2\left(k_{0}=0\right)}$ is positive, and for transparent materials $\left(k_{0} / n_{0}<10^{-4}\right), n_{2}$ is constant and equal to the value given by Eqs. (1) (so $n_{2} / n_{2\left(k_{0}=0\right)}$ $\approx 1$ ). It is also seen that the correction term introduced in this paper for absorbing materials becomes important for low values of $k_{0} / n_{0}$ when the ratio $\chi_{I}^{(3)} / \chi_{R}^{(3)}$ is large. In materials with high absorption $\left(k_{0} / n_{0} \geqslant I\right)$ the term ( $\left.1-k_{0}{ }^{2} / n_{0}^{2}\right)^{-1}$ of Eq. (18) dominates the curve, and the value of $n_{2} / n_{2\left(k_{0}=0\right)}$ shows a decreasing behavior with in-

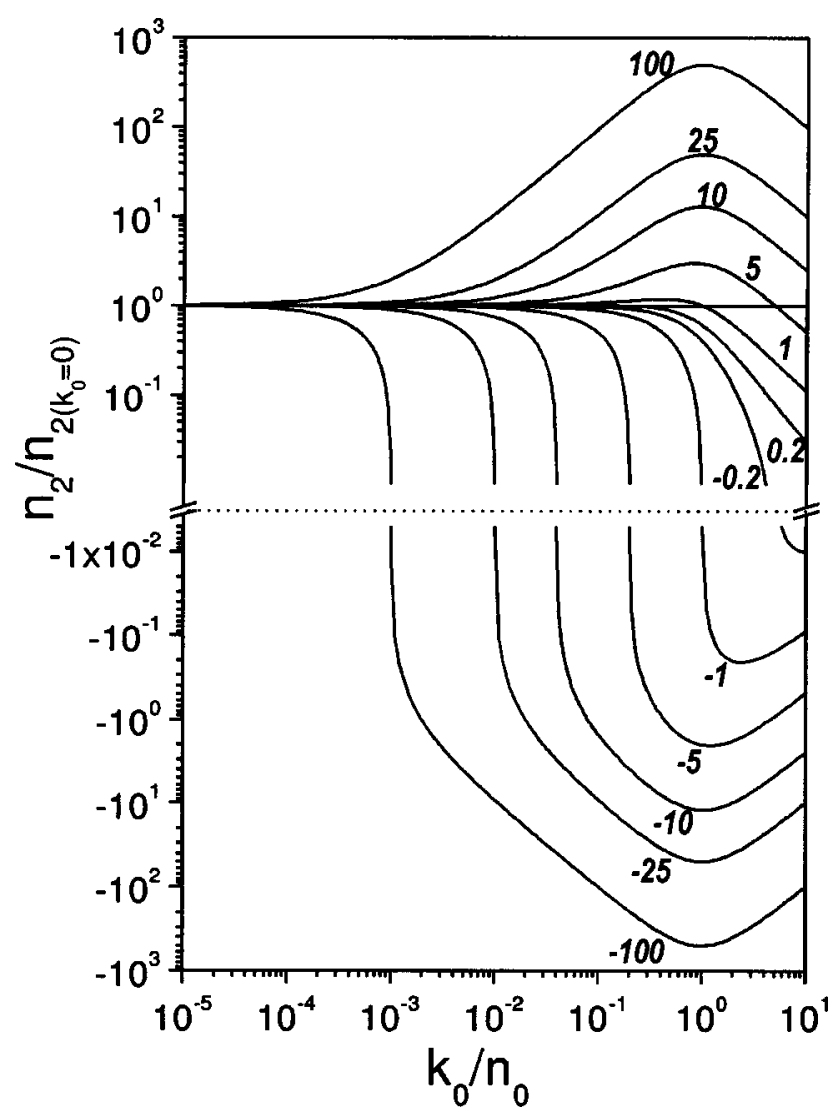

Fig. 1. Calculated $n_{2} / n_{2\left(k_{0}=0\right)}$ ratio as a function of $k_{0} / n_{0}$. The curves correspond to the different indicated values of the $\chi_{I}^{(3)} / \chi_{R}^{(3)}$ ratio.

creasing values of $k_{0} / n_{0}$. This effect is more important when the ratio $\chi_{I}^{(3)} / \chi_{R}^{(3)}$ is small, in which case the value of $n_{2}$ calculated with the new formulas can be substantially smaller than that calculated with Eqs. (1) and thus $n_{2} / n_{2\left(k_{0}=0\right)}$ can show values well below $10^{-1}$. For negative values of $\chi_{I}^{(3)} / \chi_{R}^{(3)}$, corresponding to real and imaginary parts of the third-order susceptibility having opposite signs, the correction term reduces the nonlinear refractive index $n_{2}$ that can become zero or even reverse sign.

In the case of $k_{2}$, the corrections introduced by Eq. (16) when $k_{0} \neq 0$ are symmetrical to the ones for $n_{2}$ (shown in Fig. 1), since the correction term is analogous to the one in Eq. (15) with the exchange of $\chi_{R}^{(3)}$ and $\chi_{I}^{(3)}$ and a sign change. The cancellation of nonlinear absorptive effects (and the subsequent change in sign) will occur in this case when $\chi_{R}^{(3)}$ and $\chi_{I}^{(3)}$ have the same sign.

\section{APPLICATION TO REAL MATERIALS}

From the physical point of view, the implications of expressions (15) and (16) discussed above can be considered to be hardly observable in real systems. In particular, there are not many examples of materials with a thirdorder susceptibility essentially real showing at the same time a strong absorption. However, a dielectric media heavily doped with absorbing centers, such as a laser rod doped with transition metal or rare-earth ions, should show a nonlinear refractive index (or nonlinear absorp- 
tion coefficient) which could be potentially modulated by the amount of doping (i.e., by modifying its absorption). Obviously, the interplay between $n_{2}, k_{2}, n_{0}, k_{0}$, and $\chi^{(3)}$ is much clearer in the case of strongly absorbing systems $\left(k_{0} / n_{0}>10^{-2}\right)$ such as metals, or systems with resonant nonlinearities. In the case of pure metals, although the intrinsic third-order nonlinearities are very small $\left(\chi^{(3)}\right.$ of a Drude gas is expected to be imaginary and around $\left.10^{-13} \mathrm{esu}\right),{ }^{15}$ the ratio $k_{0} / n_{0}$ can reach values of the order of 1 (and of the order of 10 as we move further towards the infrared). Equation (15) indicates that an absorbing material with expected imaginary nonlinearities, could show observable nonlinear refractive effects.

In the case of dielectrics doped with metal nanoparticles, it is relatively easy to find values of $k_{0} / n_{0}$ of the order of 1 in the vicinity of the surface plasmon resonance of the nanocomposite. In these systems, the real and imaginary parts of $\chi^{(3)}$ have been quite often determined from $n_{2}$ and $\beta$ values obtained by Z-scan. ${ }^{12}$ In our work here, expressions (15) and (16) have been used to calculate $\chi_{R}^{(3)}$ and $\chi_{I}^{(3)}$ in $\mathrm{Cu}: \mathrm{Al}_{2} \mathrm{O}_{3}$ nanocomposites in which the values of $n_{2}$ and $\beta$ were experimentally obtained from Z-scan measurements. ${ }^{16}$ These samples are used to illustrate the impact of using Eqs. (1) instead of Eqs. (13) and (14) in the case of real, strongly absorbing materials.

The samples used were synthesized by the alternatepulsed-laser-deposition technique, and consist of a film comprising 20 layers in a sandwich structure alternating a layer of $\mathrm{Cu}$ nanocrystals surrounded by an amorphous $\mathrm{Al}_{2} \mathrm{O}_{3}$ matrix with a layer of pure $\mathrm{Al}_{2} \mathrm{O}_{3}$, the total thickness of the film being approximately $110 \mathrm{~nm}$. The three samples considered here have been grown in vacuum $\left(10^{-6}\right.$ torr) with an increasing $\mathrm{Cu}$ content (from 7.7 $\times 10^{15}$ to $16 \times 10^{15}$ atoms $/ \mathrm{cm}^{2} /$ layer) that corresponds to metal volume fractions ranging from 0.17 to 0.35 , as shown in Table 1. Accordingly, they have an increasing value of the absorption coefficient at the vicinity of the surface plasmon resonance of $\mathrm{Cu}(\lambda=600 \mathrm{~nm})$. Further details on their synthesis and morphological characterization can be found elsewhere. ${ }^{17-19}$ Their linear optical properties were determined by ellipsometry and optical transmission measurements, as also described elsewhere. $^{20}$

The nonlinear refractive index $n_{2}$ and absorption $\beta$ of the samples were determined by Z-scan measurements following the experimental configuration and the theoretical framework first reported in Ref. 12. The laser pulses used in the experiment had a duration of $7 \mathrm{ps}$ at a wavelength of $600 \mathrm{~nm}$ with an energy per pulse of the order of hundreds of nanojoules and a maximum peak intensity of
$2 \mathrm{GW} / \mathrm{cm}^{2}$. They were obtained from the output of a 1-kHz optical parametric amplifier (Spectra-Physics OPA800C) after frequency doubling the output signal beam and passing it through a grating stretcher in a four-pass configuration.

The experimentally determined values of $n_{2}, \beta$, and $k_{0}$ of the samples at $600 \mathrm{~nm}$ are shown in Table 1 along with the $\mathrm{Cu}$ content per layer ${ }^{18}$ and volume fraction of the different nanocomposites. ${ }^{21}$ For all the nanocomposite samples the linear refractive index was $n_{0} \approx 2$. It can be seen in Table 1 that $n_{2}$ and $\beta$ increase with the $\mathrm{Cu}$ content. The influence of using expressions (13) and (14) instead of Eqs. (1) is illustrated in Fig. 2 where the values of $\chi_{R}^{(3)}$ and $\chi_{I}^{(3)}$ calculated with both approaches have been plotted as a function of $k_{0}$. Each point in Fig. 2 corresponds to a different sample in which the variation of the amount of $\mathrm{Cu}$ leads to a modification of the size, morphology, and concentration of the nanoparticles, giving rise to a modification of the absorption and the effective thirdorder susceptibility of the composite, as discussed elsewhere. $^{21}$ We can see in Fig. 2 that although the calculated values of $\chi_{R}^{(3)}$ are not greatly modified if one assumes negligible absorption [thus using Eqs. (1)], the magnitude and sign of $\chi_{I}^{(3)}$ are highly influenced. It is remarkable that whereas small and negative values of $\chi_{I}^{(3)}$ are obtained by assuming negligible absorption, positive

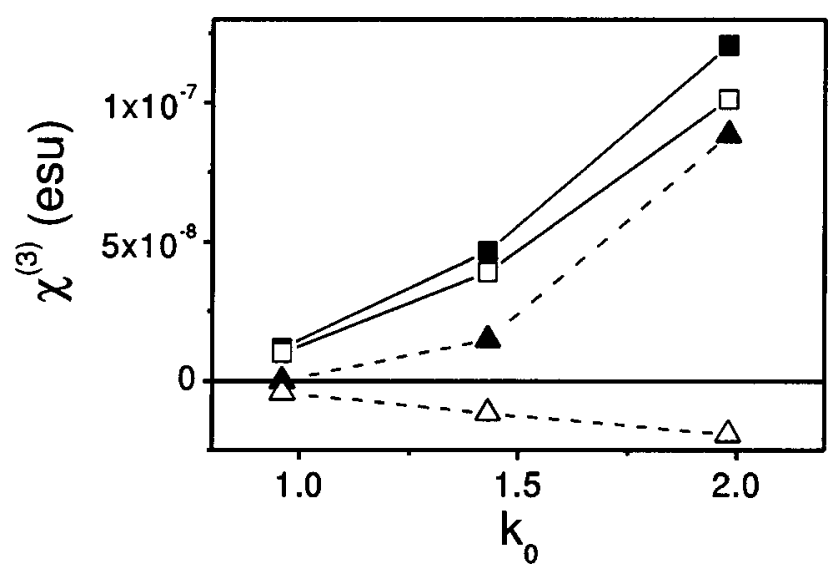

Fig. 2. Real and imaginary parts of the third-order nonlinear susceptibility of the $\mathrm{Cu}: \mathrm{Al}_{2} \mathrm{O}_{3}$ nanocomposites shown in Table 1 as a function of $\mathrm{k}_{0}$. The plotted values correspond to the determination of $\chi_{R}^{(3)}$ and $\chi_{I}^{(3)}$ from the experimental values of $n_{2}$ and $\beta$ measured by Z-scan by use of Eqs. (1)—open squares, $\chi_{R}^{(3)}$, open triangles, $\chi_{I}^{(3)}$-and by use of the correct expression for absorbing materials, Eqs. (15) and (16)—filled squares, $\chi_{R}^{(3)}$, filled triangles, $\chi_{I}^{(3)}$.

Table 1. Structural and Optical Properties of $\mathrm{Cu}: \mathrm{Al}_{2} \mathrm{O}_{3}$ Nanocomposites

\begin{tabular}{|c|c|c|c|c|}
\hline $\begin{array}{l}\text { Cu Areal } \\
\text { Content } \\
\left(\times 10^{15}\right. \\
\left.\text { atoms } / \mathrm{cm}^{2} \text { /layer }\right)\end{array}$ & $\begin{array}{l}\mathrm{Cu} \text { Volume } \\
\text { Fraction, } p\end{array}$ & $\begin{array}{c}\text { Linear } \\
\text { Absorption } \\
\text { Index, }{ }^{a} k_{0}\end{array}$ & $\begin{array}{c}\text { Nonlinear } \\
\text { Refractive } \\
\text { Index, }{ }^{\mathrm{a}} n_{2} \\
\left(10^{-10} \mathrm{~cm}^{2} / \mathrm{W}\right)\end{array}$ & $\begin{array}{c}\text { Nonlinear } \\
\text { Absorption } \\
\text { Coefficient, }{ }^{\mathrm{a}} \beta \\
\left(10^{-5} \mathrm{~cm} / \mathrm{W}\right)\end{array}$ \\
\hline 7.7 & 0.17 & 0.95 & 0.9 & 0.8 \\
\hline 11.5 & 0.25 & 1.45 & 3.2 & 2.0 \\
\hline 16 & 0.35 & 2.00 & 10 & 4.0 \\
\hline
\end{tabular}

${ }^{a}$ At $\lambda=600 \mathrm{~nm}$. 
values are determined if $\chi_{I}^{(3)}$ is rigorously calculated. This indicates that the experimentally observed saturation of absorption $(\beta<0)$ is caused actually by a positive $\chi_{I}^{(3)}$. Furthermore, for the highest value of $k_{0}$, the value of $\chi_{I}^{(3)}$ becomes comparable to that of $\chi_{R}^{(3)}$.

Unfortunately, with the existing theoretical modelsnot valid for metal volume fractions as high as that of the samples used here, as discussed in Ref. 21-it is not possible to extract the intrinsic third-order susceptibility of the metal particles from that corresponding to the composite. Therefore, we cannot correlate the obtained experimental values of $\chi_{R}^{(3)}$ and $\chi_{I}^{(3)}$ with the microscopic mechanisms responsible for the nonlinear response of our nanocomposite samples, something that is also beyond the scope of this paper. In any case, the results obtained in these metal nanocomposite samples clearly show that the use of Eqs. (1) for absorbing materials can lead to large errors in the determination of $\chi^{(3)}$.

\section{CONCLUSION}

We have obtained analytical expressions relating the real $\chi_{R}^{(3)}$ and imaginary parts $\chi_{I}^{(3)}$ of the third-order, nonlinear optical susceptibility to the nonlinear refractive index $n_{2}$ and the nonlinear absorption coefficient $\beta$ or $k_{2}$ that are valid for absorbing systems. These expressions show that in the case of nonnegligible absorption, nonlinear refractive or absorptive effects are the consequence of the interplay between the real and imaginary parts of the first- and third-order susceptibilities of the material system. This interplay gives rise, for instance, to the appearance of an observable nonlinear refractive effect in systems with a purely imaginary third-order susceptibility. Applying these expressions to a real absorbing medium, it is shown that the use of the negligible absorption assumption $\left(k_{0}=0\right)$ can lead to large errors in the determination of the sign and magnitude of $\chi_{R}^{(3)}$ and $\chi_{I}^{(3)}$ from $n_{2}$ and $\beta$.

\section{ACKNOWLEDGMENTS}

This work has been supported by the Ministerio de Ciencia y Tecnología of Spain (DPI2002-00151, TIC200203235). R. del Coso acknowledges the financial support of the Consejería de Educación de la Comunidad de Madrid and the European Social Fund (ESF). We wish to thank J. Gonzalo, C. N. Afonso, and A. Suarez-Garcia for providing the samples and for helpful discussions.

Corresponding author R. del Coso's e-mail address is rdcl@io.cfmac.csic.es.

\section{REFERENCES}

1. D. Cotter, R. J. Manning, K. J. Blow, A. D. Ellis, A. E. Kelly, D. Nesset, I. D. Phillips, A. J. Poustie, and D. C. Rogers, "Nonlinear optics for high-speed digital information processing," Science 286, 1523-1528 (1999).

2. D. W. Hall, M. A. Newhouse, N. F. Borrelli, W. H. Dumbaugh, and D. L. Weildman, "Nonlinear optical susceptibilities of high-index glasses," Appl. Phys. Lett. 54, 1293-1295 (1989).
3. N. Bloembergen, "Nonlinear optics of polymers: fundamentals and applications," J. Nonlinear Opt. Phys. Mater. 5, 1-7 (1996).

4. C. J. Hamilton, J. H. Marsh, D. C. Hutchings, J. S. Aitchison, G. T. Kennedy, and W. Sibbett, "Localized Kerr-type nonlinearities in GaAs/AlGaAs multiple-quantum-well structures at $1.55 \mu \mathrm{m}$," Appl. Phys. Lett. 68, 3078-3080 (1996).

5. G. Banfi, V. Degiorgio, and D. Ricard, "Nonlinear optical properties of semiconductor nanocrystals," Adv. Phys. 47, 447-510 (1998).

6. R. F. Haglund, Jr., C. N. Afonso, G. Battaglin, M. Godbole, F. Gonella, J. D. Hamilton, D. H. Lowndes, R. H. Magruder III, P. Mazzoldi, D. H. Osborne, Jr., and J. Solis, "Effects of laser and particle beams on the synthesis and nonlinear optical response of nanostructures," in Laser Applications in Microelectronic and Optoelectronic Manufacturing II, J. J. Dubowski, ed., Proc. SPIE 2991, 90-101 (1997).

7. Y. R. Shen, The Principles of Nonlinear Optics (Wiley, New York, 1984).

8. R. W. Boyd, Nonlinear Optics (Academic, Boston, Mass., 1992).

9. R. L. Sutherland, Handbook of Nonlinear Optics (Marcel Dekker, New York, 1996)

10. R. de Nalda, R. del Coso, J. Requejo-Isidro, J. Olivares, A. Suarez-Garcia, J. Solis, and C. N. Afonso, "Limits to the determination of the nonlinear refractive index by the Z-scan method," J. Opt. Soc. Am. B 19, 289-296 (2002).

11. R. F. Haglund, Li Yang, Lina Yang, R. Dorsinville, and R. R. Alfano, "Nonlinear optical properties of metal quantum-dot composites synthesized by ion implantation," Nucl. Instrum. Methods Phys. Res. B 91, 493-504 (1994).

12. M. Sheik-Bahae, A. A. Said, T. Wei, D. J. Hagan, and E. W. Van Stryland, "Sensitive measurement of optical nonlinearities using a single beam," IEEE J. Quantum Electron. 26, 760-769 (1990).

13. S. M. Lima, H. Jiao, L. A. O. Nunes, and T. Catanuda, "Nonlinear refraction spectroscopy in resonance with laser lines in solids," Opt. Lett. 27, 845-847 (2002).

14. E. Van Keuren, T. Wakebe, R. Andreaus, H. Mohwald, W. Schrof, V. Belov, H. Matsuda, and R. Rangel-Rojo, "Wavelength dependence of the third-order nonlinear optical properties of polythiophene-selenophene derivative film," Appl. Phys. Lett. 75, 3312-3314 (1999).

15. F. Hache, D. Ricard, C. Flytzanis, and U. Kreibig, "The optical Kerr effect in small metal particles and metal colloids: the case of gold," Appl. Phys. A 47, 347-357 (1988).

16. R. del Coso, J. Solis, J. Gonzalo, and C. N. Afonso, "Ultrafast temporal response of the third order nonlinear effects in $\mathrm{Cu}: \mathrm{Al}_{2} \mathrm{O}_{3}$ nanocomposites" (manuscript available from authors on request).

17. R. Serna, C. N. Afonso, J. M. Ballesteros, A. Naudon, D. Babonneau, and A. K. Petford-Long, "Size, shape anisotropy, and distribution of $\mathrm{Cu}$ nanocrystals prepared by pulsed laser deposition," Appl. Surf. Sci. 138-139, 1-5 (1999).

18. C. N. Afonso, J. Gonzalo, R. Serna, J. C. G. de Sande, C. Ricolleau, C. Grigis, M. Gandais, D. E. Hole, and P. D. Townsend, "Vacuum versus gas environment for the synthesis of nanocomposite films by pulsed laser deposition," Appl. Phys. A 69, S201-S207 (1999).

19. R. Serna, J. Gonzalo, A. Suárez-García, C. N. Afonso, J. P. Barnes, A. K. Petford-Long, R. C. Doole, and D. Hole, "Structural studies of pulsed-laser deposition, nanocomposite, metal-oxide films," J. Microsc. 201, 250-255 (2001).

20. A. Suarez-Garcia, R. del Coso, R. Serna, J. Solis, and C. N Afonso, "Controlling the transmission at the surface plasmon resonance of nanocomposite films using photonic structures," Appl. Phys. Lett. 83, 1842-1844 (2003)

21. R. del Coso, J. Requejo-Isidro, J. Solis, J. Gonzalo, and C. N. Afonso, "Third order nonlinear optical susceptibility of $\mathrm{Cu}: \mathrm{Al}_{2} \mathrm{O}_{3}$ nanocomposites: from spherical nanoparticles to the percolation threshold," J. Appl. Phys. (to be published). 